clonal $\mathrm{T}$ cell proliferation in two patients after gene therapy for SCID-X1. Science. 302:415-419.

7. Schroder, A.R., et al. 2002. HIV-1 integration in the human genome favors active genes and local hotspots. Cell. 110:521-529.

8. Wu, T., et al. 2000. Prolonged high-level detection of retrovirally marked hematopoietic cells in nonhuman primates after transduction of CD34+ progenitors using clinically feasible methods. Mol. Ther. 1:285-293.

9. Hematti, P., et al. 2004. Distinct genomic integration of MLV and SIV vectors in primate hematopoietic stem and progenitor cells. PLoS Biol. 2:e423.

10. Adjali, O., et al. 2005. In vivo correction of ZAP-70 immunodeficiency by intrathymic gene transfer. J. Clin. Invest. 115:2287-2295. doi:10.1172/JCI23966. 11. Elder, M.E. 1998. ZAP-70 and defects of T-cell receptor signaling. Semin. Hematol. 35:310-320.

12. Wiest, D.L., et al. 1997. A spontaneously arising mutation in the DLAARN motif of murine ZAP70 abrogates kinase activity and arrests thymocyte development. Immunity. 6:663-671.

13. Chan, A.C., et al. 1994. Differential expression of ZAP-70 and Syk protein tyrosine kinases, and the role of this family of protein tyrosine kinases in TCR signaling. J. Immunol. 152:4758-4766.

14. Habibian, H.K., et al. 1998. The fluctuating phenotype of the lymphohematopoietic stem cell with cell cycle transit. J. Exp. Med. 188:393-398.

15. Takatoku, M., et al. 2001. Avoidance of stimulation improves engraftment of cultured and retrovirally transduced hematopoietic cells in primates. J. Clin. Invest. 108:447-455. doi:10.1172/JCI200112593.

16. Sauce, D., et al. 2002. Retrovirus-mediated gene transfer in primary $\mathrm{T}$ lymphocytes impairs their anti-Epstein-Barr virus potential through both culture-dependent and selection process-dependent mechanisms. Blood. 99:1165-1173.

17. Marodon, G., and Klatzmann, D. 2004. In situ transduction of stromal cells and thymocytes upon intrathymic injection of lentiviral vectors. BMC Immunol. 5:18. http://www.biomedcentral. com/1471-2172/5/18.

18. Buckley, R.H. 2004. Molecular defects in human severe combined immunodeficiency and approaches to immune reconstitution. Annu. Rev. Immunol. 22:625-655.

19. Stephan, V., et al. 1996. Atypical X-linked severe combined immunodeficiency due to possible spontaneous reversion of the genetic defect in T cells. N. Engl. J. Med. 335:1563-1567.

\title{
Spring brings breezes, wheezes, and pollen oxidases
}

\author{
Darren R. Ritsick and J. David Lambeth
}

Department of Pathology and Laboratory Medicine, Emory University School of Medicine, Atlanta, Georgia, USA.

\begin{abstract}
While the release of pollen into the air is essential for the reproduction of plants, the accidental yet inevitable uptake of pollen into human airways can cause symptoms of seasonal allergies and asthma. The symptomatic response to pollen is caused by granulocytes that produce inflammation, which is due in part to oxidative stress through the action of NADPH oxidases. The recruitment of these inflammatory granulocytes was previously thought to depend entirely on the activation of an adaptive immune response. In this issue of the JCI, Boldogh et al. demonstrate that pollens contain endogenous NADPH oxidase activity, which functions to generate local "danger signals" in nearby airway epithelium (see the related article beginning on page 2169). These signals in turn trigger the early recruitment of granulocytes, even in the absence of the adaptive immune response. These findings suggest that inhibition of the pollen oxidase may provide a way to antagonize allergic inflammation at a very early step.
\end{abstract}

Spring... in the space of a few weeks, the drab winter grays give way to a riot of reds, pinks, yellows, and whites, as azaleas, dogwoods, and a host of other plants celebrate the arrival of longer, hotter days. But for many individuals, the bright banners of spring signal not a celebration, but a feeling of dread that augurs frequent trips to the pharmacy for the latest over-thecounter remedies. For amidst the color, another riot is taking place: in a reproductive frenzy, each member of the plant world is busily packaging its genome into protective capsules, which are ejected into the atmosphere or hitch rides on the bodies of insects. While a pollen count of 120

Conflict of interest: The authors have declared that no conflict of interest exists.

Citation for this article: J. Clin. Invest. 115:2067-2069 (2005). doi:10.1172/JCI26023. particles per cubic meter is considered high in many parts of the country, in the pollen belt of the southern US, counts of 6,000 are not unusual, and the accumulated particles confer a distinctly yellowish cast to the atmosphere.

While Mother Nature is merely assuring the reproductive success of her immobile plant kingdom by sowing the atmosphere with these tiny portable packages of DNA, the inadvertent consequence is that many of these particles do not find their intended targets but end up in the airways and mucous membranes of hapless humans. It is no wonder that the sounds of spring are punctuated by sneezes and coughs, and that the spring colors are seen through the blur of watery, itchy eyes. Indeed, as many as 35 million people in the US suffer from allergic reactions to pollens, ranging from annoying allergic rhinitis and allergic conjunctivitis to the more dangerous allergic asthma (1).

According to established paradigms, the pollen grains are interpreted by the host as invading foreign bodies, which results in a defensive immune reaction against the proteins and other biomolecules on the pollen's surface (2). This reaction involves inflammatory cells, including granulocytes such as eosinophils and mast cells, and can lead to chronic inflammation, airway hyperresponsiveness, bronchial constriction, increased fluid and mucus secretion, and in some cases, life-threatening sequelae (3). According to this conventional model, the inflammatory response includes the production of ROS via a membrane-associated superoxide-generating NADPH oxidase that is present in granulocytes (4, $5)$. This oxidase consists of the membranebound flavocytochrome $b_{558}$, of which gp91phox is the catalytic subunit, plus several regulatory subunits. When activated, the oxidase catalyzes the transfer of an electron from NADPH to molecular oxygen and forms superoxide $\left(\mathrm{O}_{2}{ }^{\circ}\right)$, with secondary production of hydrogen peroxide and other chemically reactive species (6, 7). Collectively, ROS can produce a wide range of molecular damage, which results in the oxidative modification of a variety of biomolecules as well as cellular damage and disruption of membrane integrity. In addition, ROS have more recently been implicated as intra- and intercellular signal molecules and therefore may participate 


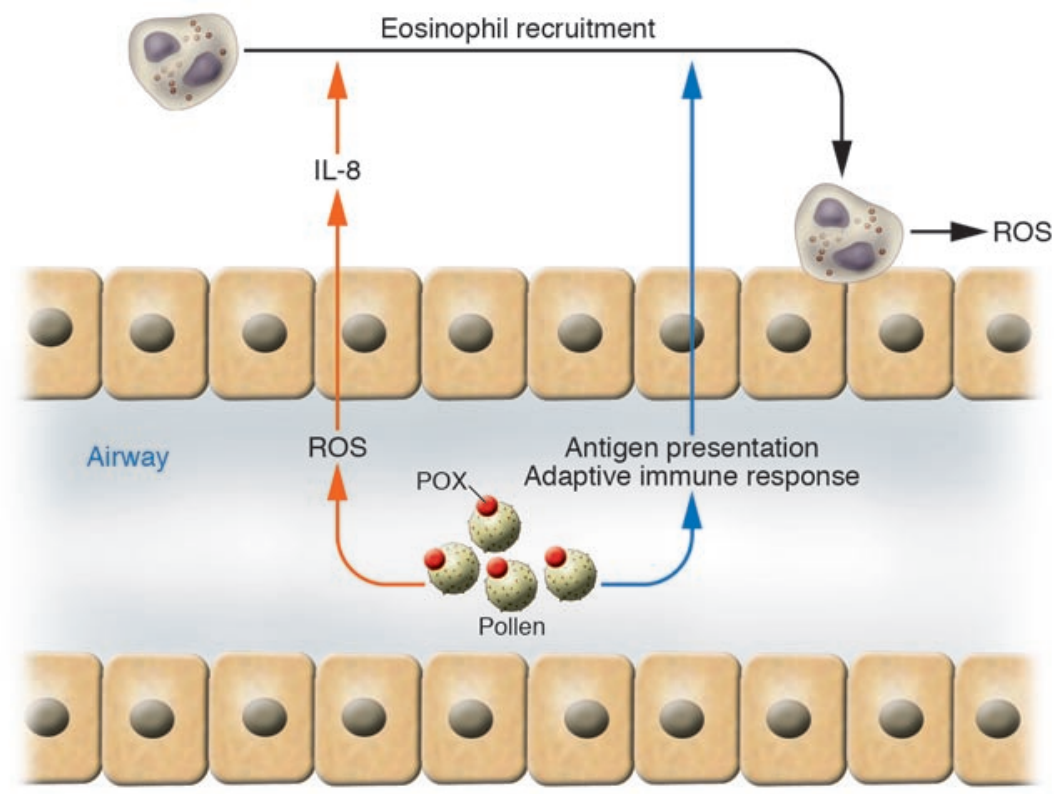

Figure 1

A binary model for pollen-induced inflammation. Pollen triggers an adaptive immune response through the process of antigen presentation by professional antigen-presenting cells. This response also triggers recruitment of granulocytes including eosinophils and results in inflammation, which is due in part to the production of ROS by the eosinophil NADPH oxidase. In this issue of the $\mathrm{JCl}$, Boldogh and colleagues (9) demonstrate that pollen has its own NADPH oxidase activity (referred to here as pollen oxidase, POX) that induces local oxidative stress and IL-8 secretion and triggers early recruitment of eosinophils even in the total absence of an adaptive immune response.

more directly as mediators of the inflammatory response (8).

According to this conventional view, if pollen acts passively to initiate the allergic reaction through its antigenic properties, then it is the host cells, including the granulocytes, that are wreaking havoc by overreacting to the pollen. However, in this issue of the JCI, Boldogh and colleagues provide evidence that pollen itself is playing an active role in triggering aspects of the host allergic response and perhaps directly damaging host cells (9). This active role depends, ironically enough, on pollen NADPH oxidase activity, the same enzymatic activity that eosinophils trigger upon exposure to the invading pollen. According to Boldogh et al., pollen's endogenous ROS-generating activity can expose airway cells to ROS prior to the recruitment of inflammatory cells. The authors further conclude that pollen NADPH oxidase activity is involved in the recruitment of inflammatory cells, possibly through induction of IL-8 by p38 MAPK, but alone is not sufficient for such recruitment. These surprising findings represent a departure from the previous concept that ROS are derived exclusively from host immune cells during an allergic response and that their recruitment is dependent upon prior induction of an adaptive immune response.

Based on the present study, the authors propose a binary model for the induction of an inflammatory response by pollen (9). Their model proposes that pollen NADPH oxidases provide a signal independent of the adaptive immune response that combines with the pollen antigen-induced adaptive immune response to induce allergic airway inflammation (Figure 1). In this model, oxidative stress produced by pollen NADPH oxidases provides an acute "danger signal" to the surrounding mucosa that triggers an initial influx of innate immune cells. Thus, the authors demonstrate for the first time to our knowledge that pollens produce ROS and that such production contributes to the activation of an immune response.

\section{NADPH oxidases in physiology and pathology}

Although the pollen NADPH oxidase in question (9) has not yet been genetically identified, its properties place it very like- ly within a growing family of gp91phox homologs, the Nox/Duox family (10-12). While characterized initially in neutrophils and macrophages, it is now clear that related NADPH oxidases are expressed in a variety of eukaryotic tissues and in species of plants, insects, nematodes, amoeba, and vertebrates. In animals, their biological roles have been defined only in a small number of cases such as the well-documented role of the phagocyte NADPH oxidase in host defense (13), the role of Nox3 in otolith biogenesis in the inner ear (14, $15)$, and the role of Duox 2 in thyroid hormone biosynthesis (16). In addition, there is growing evidence in support of a role for these enzymes in generating ROS that serve as diffusible signals required for a variety of cellular functions. Indeed, ROS have been implicated in signaling apoptosis, mitogenic responses, angiogenesis, smooth muscle hypertrophy, differentiation, and immune signaling. In plants, NADPH oxidases function in host defense (17), stomatal closure (18), and root hair tip growth where they play a role in polarized cell growth (19). Root hairs are 1 of 2 highly differentiated tip growing structures in plants, the other of which are pollen tubes (20). Given the analogous polarized growth characteristics of the 2 cell types, it seems plausible that pollen tubes also contain an NADPH oxidase that may participate in polarized growth. Thus the NADPH oxidase of pollen may be a developmental holdover derived from the cell of origin. Alternatively, as suggested by Boldogh et al. (9), seed germination requires ROS; it is possible that ROS is also required for pollen germination.

Despite whatever advantage it confers for the plant, when the pollen oxidase comes into contact with the human airway, pathological responses occur. This is not entirely surprising, given that ROS have long been documented to cause molecular damage and aberrant signaling, collectively referred to as oxidative stress. In this regard, the discovery of a pathogenic role for pollen NADPH oxidase in the allergic response adds to a growing list of proposed pathological roles for members of this family of enzymes. While the function of these enzymes is likely to be necessary for maintenance of normal signaling, abnormal expression or function of Nox/Duox enzymes has been proposed to be involved in the pathogenesis of hypertension, atherosclerosis, diabetes, and cancer. The work by Boldogh et al. (9) implies that drugs designed to inhibit the pollen NADPH oxi- 
dase may provide a new therapeutic avenue to block allergic inflammation at its earliest known step and help those of us who live in the pollen belt to better appreciate the arrival of spring.

Address correspondence to: J. David Lambeth, Department of Pathology and Laboratory Medicine, 615 Michael Street, Emory University School of Medicine, Atlanta, Georgia 30322, USA. Phone: (404) 727-5875; Fax: (404) 727-2783; E-mail: noxdoc@mac.com.

1. National Institute of Allergy and Infectious Disease. 2003. Airborne allergens: something in the air. National Institutes of Health. NIH Publication No. 03-7045. http://www.niaid.nih.gov/publications/allergens/airborne_allergens.pdf.

2. Herrick, C.A., and Bottomly, K. 2003. To respond or not to respond: T cells in allergic asthma. Nat. Rev. Immunol. 3:405-412.
3. Bousquet, J., et al. 1990. Eosinophilic inflammation in asthma. N. Engl. J. Med. 323:1033-1039.

4. Bowler, R.P., and Crapo, J.D. 2002. Oxidative stress in allergic respiratory diseases. J. Allergy Clin. Immunol. 110:349-356.

5. Andreadis, A.A., Hazen, S.L., Comhair, S.A.A., and Erzurum, S.C. 2003. Oxidative and nitrosative events in asthma. Free Radic. Biol. Med. 35:213-225.

6. Babior, B.M., Lambeth, J.D., and Nauseef, W. 2002. The neutrophil NADPH oxidase. Arch. Biochem. Biophys. 397:342-344.

7. Vignais, P.V. 2002. The superoxide-generating NADPH oxidase: structural aspects and activation mechanism. Cell. Mol. Life Sci. 59:1428-1459.

8. Lambeth, J.D. 2002. Nox/Duox family of nicotinamide adenine dinucleotide (phosphate) oxidases. Curr. Opin. Hematol. 9:11-17.

9. Boldogh, I., et al. 2005. ROS generated by pollen NADPH oxidase provide a signal that augments antigen-induced allergic airway inflammation. J. Clin. Invest. 115:2169-2179. doi:10.1172/JCI24422.

10. Suh, Y.A., et al. 1999. Cell transformation by the superoxide-generating oxidase Mox1. Nature. 401:79-82.

11. Lambeth, J.D., Cheng, G., Arnold, R.S., and Edens, W.A. 2000. Novel homologs of gp91phox. Trends Biochem. Sci. 25:459-461.
12. Lambeth, J.D. 2004. NOX enzymes and the biology of reactive oxygen. Nat. Rev. Immunol. 4:181-189.

13. Heyworth, P.G., Cross, A.R., and Curnutte, J.T. 2003. Chronic granulomatous disease. Curr. Opin. Immunol. 15:578-584.

14. Banfi, B., et al. 2004. NOX3, a superoxide-generating NADPH oxidase of the inner ear. J. Biol. Chem. 279:46065-46072.

15. Paffenholz, R., et al. 2004. Vestibular defects in headtilt mice result from mutations in Nox3, encoding an NADPH oxidase. Genes Dev. 18:486-491.

16. Moreno, J.C., et al. 2002. Inactivating mutations in the gene for thyroid oxidase 2 (THOX2) and congenital hypothyroidism. N. Engl. J. Med. 347:95-102.

17. Lamb, C., and Dixon, R.A. 1997. The oxidative burst in plant disease resistance. Annu. Rev. Plant Physiol. Plant Mol. Biol. 48:251-275.

18. Kwak, J.M., et al. 2003. NADPH oxidase AtrbohD and AtrbohF genes function in ROS-dependent ABA signaling in Arabidopsis. EMBO J. 22:2623-2633.

19. Foreman, J., et al. 2003. Reactive oxygen species produced by NADPH oxidase regulate plant cell growth. Nature. 422:442-446.

20. Feijo, J.A., Costa, S.S., Prado, A.M., Becker, J.D., and Certal, A.C. 2004. Signalling by tips. Curr. Opin. Plant Biol. 7:589-598.

\section{Insulin infusion in acute illness}

\section{Paresh Dandona, Priya Mohanty, Ajay Chaudhuri, Rajesh Garg, and Ahmad Aljada}

Division of Endocrinology, Diabetes and Metabolism, State University of New York at Buffalo, and Kaleida Health, Buffalo, New York, New York, USA.

\begin{abstract}
The discovery of the antiinflammatory effect of insulin and the proinflammatory effect of glucose has not only provided novel insight into the mechanisms underlying several disease states but has also provided a rationale for the treatment of hyperglycemia in several acute clinical conditions. Van den Berghe et al. previously showed the benefits of intensive glycemic control with insulin in patients admitted to intensive care units. In this issue of the JCI, the same group of investigators now demonstrates that infusion of insulin to restore euglycemia in these patients results in a marked reduction in inflammatory indices such as adhesion molecules, hepatic iNOS, and plasma NO metabolites (see the related article beginning on page 2277). The reduction in the mediators of inflammation may thus be responsible for the impressive improvement in clinical outcomes following insulin therapy, and the results suggest a new paradigm in which glucose and insulin are related not only through their metabolic actions but also through their opposite effects on inflammatory mechanisms.
\end{abstract}

The importance of hyperglycemia in the pathogenesis and prognosis of acute illness and the benefit of controlling glucose with insulin in the critically ill hospitalized patient have emerged over the past 4 years. Three sets of data appeared almost simultaneously, which drew attention to this issue.

Nonstandard abbreviations used: AP-1, activator protein-1; EGR-1, early growth response-1; ICU, intensive care unit; TF, tissue factor.

Conflict of interest: Paresh Dandona is a consultant and speaker for Sanofi-Aventis and Novo Nordisk.

Citation for this article: J. Clin. Invest. 115:2069-2072 (2005). doi:10.1172/JCI26045.
First, glucose and lipid administration were shown to result in oxidative and inflammatory stress (1-3). Second, insulin, the hormone secreted in response to glucose and macronutrient intake, was found to suppress ROS generation and the activation of inflammatory mechanisms $(4,5)$. Thus, glucose was shown to be proinflammatory while insulin is antiinflammatory (Figure 1). Third, and almost simultaneously with the 2 previous studies, came the observation that the restoration of normoglycemia by insulin infusion in hyperglycemic patients in a surgical intensive care unit (ICU) resulted in a $50 \%$ reduction in mortality along with several other benefits, including a reduction in the incidence of renal failure (and thus the need for dialysis), septicemia, and ICU neuropathy (6). The need for blood transfusion and the duration of ventilation also fell significantly in these patients. These effects of insulin infusion in acutely ill patients were remarkable and totally unexpected. This large, landmark study provided clinical evidence that the proinflammatory effects of glucose and antiinflammatory effects of insulin probably play an important role in determining clinical outcome in acute illness. In this same study (6), the clinical benefits were associated with a fall in C-reactive protein level (7). A close relationship was found between the fall in blood glucose concentration and the improvement in clinical outcome.

Overall, the data emphasized that hyperglycemia is potentially toxic (6). This is consistent with a proinflammatory effect of glucose. In a subsequent publication, it was shown that there were marked mitochondrial abnormalities in the hepatocytes of the control group, which were prevented in the insulin infusion group with the restoration of normoglycemia (8). The mitochondrial abnormalities were char- 